\title{
EFEITO DA ADIÇÃO DE EXTRATO DE ALECRIM, CHÁ VERDE E ÓLEO DE LINHAÇA SOBRE A ESTABILIDADE OXIDATIVA, PROPRIEDADES FÍSICO- QUÍMICAS E SENSORIAIS DE HAMBÚRGUER BOVINO
}

\author{
ANA KAROLINE FERREIRA IGNÁCIO CÂMARA* \\ GRACIELA FUJIMOTO** \\ MARIA CECÍLIA ENES RIBEIRO** \\ DAIANA NOVELLO*** \\ MARISE APARECIDA RODRIGUES POLLONIO****
}

\begin{abstract}
Os efeitos da adição de extrato de alecrim e chá verde e substituição da gordura vegetal por óleo de linhaça dourada nas propriedades físico-químicas e sensoriais de hambúrguer bovino foram estudados. Utilizou-se o método de TBARS para avaliação da estabilidade oxidativa e teste de aceitação na avaliação sensorial, além da realização de análises físico-químicas de determinação objetiva da cor, lipídios, pH, atividade de água, perdas por cocção e encolhimento. A adição de óleo de linhaça influenciou o aumento da intensidade da coloração amarela em níveis acima de 2,5\%. A avaliação sensorial indicou melhor aceitação de F2, por painel sensorial não treinado. Não houve diferenças significativas $(p<0,05)$ entre a aparência e aroma dos produtos. Os antioxidantes presentes e níveis de gordura e óleo influenciaram de modo semelhante o atributo sabor na percepção dos consumidores. Avaliando-se a atividade antioxidante dos extratos vegetais pelo índice de TBARS, foi verificado melhor desempenho do eritorbato de sódio em relação aos extratos vegetais. Entre os extratos vegetais constatouse que para a concentração de $2,5 \%$ de óleo de linhaça, ambos apresentaram semelhanças na atividade antioxidante. Os valores de gorduras totais foram consideravelmente reduzidos (redução de $61,5 \%$ ) e o perfil lipídico foi melhorado com o acréscimo de ácidos graxos polinsaturados. A estratégia de reformulação descrita, com base na substituição da gordura vegetal por óleo de linhaça dourada e antioxidantes naturais em hambúrguer bovino demonstrou ser tecnologicamente viável e uma alternativa de consumo de produtos cárneos mais saudáveis.
\end{abstract}

PALAVRAS-CHAVE: HAMBÚRGUER; ÓLEO DE LINHAÇA; OXIDAÇÃO LIPÍDICA; CHÁ VERDE; ALECRIM.

*Mestre em Tecnologia de Alimentos, Professora, Departamento de Engenharia de Alimentos, Universidade Federal de São João Del Rei, UFSJ, Sete Lagoas, MG, Brasil (e-mail: anakarolineign@gmail.com).

**Doutorandas em Tecnologia de Alimentos, Faculdade de Engenharia de Alimentos, Universidade Estadual de Campinas, UNICAMP, Campinas, SP, Brasil (e-mail: cissaribeiro@gmail.com; gracielafujimoto@gmail. com).

*** Doutora em Tecnologia de Alimentos, Professora, Faculdade de Nutrição, Universidade Estadual do CentroOeste, UNICENTRO, Guarapuava, PR, Brasil (e-mail: nutridai@yahoo.com.br).

**** Doutora em Tecnologia de Alimentos, Professora, Faculdade de Engenharia de Alimentos, Departamento de Tecnologia de Alimentos, Universidade Estadual de Campinas, UNICAMP, Campinas, SP, Brasil (e-mail: marise@fea.unicamp.br). 


\section{INTRODUÇÃO}

A indústria de carnes, assim como outros setores da área de alimentos, é alvo de importantes transformações, conseqüência das inovações tecnológicas e das mudanças exigidas pelos consumidores. Uma das principais tendências que marca a evolução do consumo de produtos cárneos surge da preocupação dos consumidores com a saúde, favorecida pelas novas recomendações e orientações nutricionais aconselhadas por diversas instituições (JIMÉNEZCOLMENERO, 2006).

A American Heart Association e outras organizações de saúde têm recomendado diminuir o consumo diário de gordura e colesterol como uma forma de prevenção ao desenvolvimento de doenças cardiovasculares. Tais recomendações detalhadamente relatam que apenas $30 \%$ do total de calorias da dieta sejam provenientes das gorduras, sendo que o consumo de gorduras saturadas não deve ultrapassar $10 \%$ deste total, e a ingestão de colesterol esteja abaixo de $300 \mathrm{mg}$ por dia (OLMEDILLA-ALONSO, JIMÉNEZ-COLMENERO e SÁNCHEZ-MUNIZ, 2013; WHO, 2003).

Porém, gorduras desempenham papel fundamental nos aspectos sensoriais e de funcionalidade em vários produtos alimentícios. A gordura interage com outros ingredientes para desenvolver textura e auxilia de uma forma geral nas propriedades de plasticidade e consistência dos alimentos (GIESE, 1992). Em função dessas propriedades, um dos maiores desafios tecnológicos em produtos cárneos é a substituição da gordura por compostos mais saudáveis.

Várias pesquisas têm estudado estratégias para reduzir a gordura saturada utilizada em produtos cárneos. Desta maneira, algumas tentativas incluem o uso de óleos vegetais como substitutos desta gordura, proporcionando aos produtos um melhor perfil de ácidos graxos e menores níveis de colesterol quando comparados com os tradicionais (ÖZVURAL e VURAL, 2008; VALENCIA, ANSORENA e ASTIASARÁN, 2006). Yilmaz, Simsek e Isikli (2002) estudaram o efeito da substituição da gordura animal por sete formulações diferentes em salsichas, dentre elas, uma com óleo de girassol. A salsicha produzida com óleo de girassol obteve resultados positivos em relação ao conteúdo de ácidos graxos essenciais e insaturados e em relação às características sensoriais.

Alguns óleos vegetais possuem altas quantidades de ácidos graxos monoinsaturados (MUFA's) e polinsaturados (PUFA's), os quais podem auxiliar na diminuição da lipoproteína de baixa densidade no plasma (LDL). Além disso, níveis maiores de ácidos graxos polinsaturados podem promover um aumento da lipoproteína de alta densidade (HDL) que auxilia na redução da incidência de doenças coronarianas (MOZAFFARIAN et al., 2005). Dentre os óleos vegetais, o óleo de linhaça tem se destacado por ser rico em PUFA's, em especial o ácido a-linolênico que tem sido bastante utilizado no enriquecimento de diversos alimentos em virtude das suas propriedades funcionais (ANSORENA e ASTIASARÁN, 2004; TREVINO et al., 2000).

Contudo, o enriquecimento de produtos cárneos com ácidos graxos insaturados pode causar uma alta susceptibilidade à oxidação lipídica, o que é um dos principais fatores limitantes da qualidade e aceitabilidade de carnes e derivados. A oxidação lipídica é uma das maiores causas de perda de qualidade em produtos cárneos processados, causando um efeito adverso no sabor, na cor e textura, assim como no valor nutricional. Além disso, provoca a degradação de vitaminas lipossolúveis e de ácidos graxos essenciais, assim como interfere na integridade e segurança do alimento, por meio da formação de compostos tóxicos, como o malonaldeído e óxidos de colesterol (ANSORENA e ASTIASARÁN, 2004; MORRISSEY et al, 1998).

Em carnes cozidas e estocadas estas reações causam off-flavors, como o sabor de requentado, denominado do termo em inglês warmed over flavour (WOF) (GRAY et al., 1996). Este processo é atribuído a auto-oxidação de compostos lipídicos da carne que geram compostos hidroperóxidos que por diferentes vias formam uma grande quantidade de compostos voláteis (JAYATHILAKAN et al., 2007). O desenvolvimento de WOF é bastante comum em carnes cozidas refrigeradas e os principais fatores que contribuem para este processo são a presença de fosfolipídeos 
e teores mais elevados de ácidos graxos insaturados (BYRNE et al., 2001; JAYATHILAKAN et al., 2007).

No desenvolvimento do WOF, os fosfolipídeos liberam ferro hemínico e não hemínico pelo rompimento da membrana, o que contribui, em conjunto com a liberação dos compostos voláteis e com as reações entre proteínas para a formação de aromas indesejáveis de ranço, perda de sabor e deterioração das características sensoriais da carne (BYRNE et al., 2003; GRÜN et al., 2006). Esta perda de qualidade torna o produto inaceitável do ponto de vista sensorial e ocorre especialmente em produtos cárneos cozidos e não curados, como hambúrgueres, almôndegas e produtos reestruturados empanados (BYRNE et al., 2001; O'SULLIVAN et al., 2003).

Diferentes estratégias podem ser adotadas para minimizar a oxidação, como por exemplo, o uso de antioxidantes (RUSAK et al., 2008) e em especial como recente tendência de reformulação em produtos cárneos, a aplicação de antioxidantes naturais. Dentre os extratos vegetais que apresentam atividade antioxidante estão os extratos de alecrim e chá verde (GEORGANTELIS et al., 2007; SEBRANEK et al., 2005).

O alecrim é uma erva aromática da família Lamiaceae com reconhecidas propriedades antioxidantes e tem sido bastante investigado para aplicação em produtos cárneos. A atividade antioxidante do alecrim tem sido atribuída à sua composição fenólica composta especialmente por diterpenos, rosmanol, rosmaridifenol, carnasol, os ácidos rosmarínico e caféico (FERNANDEZLOPEZ et al., 2005; MARIUTTI e BRAGAGNOLO, 2007). Em alguns estudos realizados em produtos cárneos, como salsichas e almôndegas, o extrato de alecrim demonstrou maior atividade antioxidante quando comparado a diversos antioxidantes naturais, como a quitosana, o $\alpha$-tocoferol, os extratos de limão e laranja, e também apresentou atividade semelhante ao BHT e BHA em salsichas cozidas, quando adicionado na concentração de 2500 ppm (SEBRANEK et al, 2005; FERNANDEZ-LOPEZ et al., 2005; GEORGANTELIS et al., 2007).

O chá verde apresenta grande riqueza fenólica composta especialmente por flavonóides e catequinas, aos quais são atribuídas atividades antioxidantes, por serem "sequestrantes" de compostos quelantes e por inibirem a lipoperoxidação (ALMAJANO et al., 2008; HORZIC et al., 2009). Em estudos conduzidos por Bozkurt (2006) foi demonstrado que o chá verde possui maior atividade antioxidante que o BHT em um embutido fermentado.

Sabendo-se que o hambúrguer é um produto cárneo industrializado com alto teor de gordura saturada e sendo o seu consumo bastante difundido na sociedade, o presente trabalho teve como objetivo avaliar a capacidade antioxidante dos extratos de alecrim e chá verde e propriedades físico-químicas e sensoriais do hambúrguer bovino com perfil lipídico modificado pela substituição da gordura vegetal pelo óleo de linhaça dourada.

\section{MATERIAL E MÉTODOS}

\subsection{MATERIAIS}

Os hambúrgueres foram preparados utilizando carne bovina M. trapezius cervicis e Tríceps brachii que correspondem respectivamente aos cortes cárneos de acém e paleta, obtidas a partir de um fornecedor industrial (JBS S.A, Brasil) e estocados no Laboratório de Carnes e Processos da Universidade Estadual de Campinas (UNICAMP) entre 1 a $4^{\circ} \mathrm{C}$ até o processamento. A preparação das matérias-primas consistiu na remoção de gordura e aponeuroses aparentes. Outros ingredientes e aditivos usados foram cloreto de sódio (Cisne, S.A. Rio de Janeiro, Brasil), maltodextrina (Corn Products, São Paulo, Brasil), alho e cebola em pó (Ibrac, São Paulo, Brasil), carragena (New Max Industrial, Americana, Brasil), gordura vegetal (Bunge, Jaguaré, Brasil), tripolifosfato de sódio e eritorbato de sódio fornecidos pela ISP Germinal, São Paulo, Brasil. O óleo de linhaça dourada foi obtido de um fornecedor especializado da cidade de Campinas (SP). A amostra de extrato natural de alecrim (GUARDIAN ${ }^{\mathrm{TM}}$ 09) foi fornecida pela empresa DuPont ${ }^{\mathrm{TM}}$ Danisco (Barueri, Brasil) e a de 
extrato de chá verde fornecida pela empresa Tovani Benzaquen Ingredientes (São Paulo, Brasil).

\subsection{TRATAMENTOS}

Foram preparadas seis formulações de hambúrguer, sendo que duas delas foram consideradas formulações controle sem adição de óleo de linhaça, com $5 \%$ de gordura vegetal: uma formulação (FE) com $0,05 \%$ de eritorbato de sódio e outra (FP) sem adição de antioxidantes. Em duas formulações denominadas F1 e F2 foram adicionados 2,5\% de óleo de linhaça e 0,05\% de extrato de alecrim e chá verde respectivamente. Nas outras duas formulações denominadas F3 e F4 foram adicionados $5 \%$ de óleo de linhaça e $0,05 \%$ de extrato de alecrim e chá verde respectivamente. As formulações destes tratamentos são demonstradas na Tabela 1. Os seguintes ingredientes e/ou aditivos também foram adicionados em percentual (\%) em cada um dos tratamentos: maltodextrina, 2,8; condimentos, 0,6; carragena, 0,2 e cloreto de sódio, 1,5 .

\section{TABELA 1- FORMULAÇÕES DE HAMBÚRGUERES BOVINOS ADICIONADOS DE ÓLEO DE LINHAÇA DOURADA, GORDURA VEGETAL E ANTIOXIDANTES}

\begin{tabular}{ccccccc}
\hline \multirow{2}{*}{ Ingredientes } & \multicolumn{7}{c}{ Tratamentos (\%) } \\
\cline { 2 - 7 } & FP & FE & F1 & F2 & F3 & F4 \\
\hline Acém & 37,45 & 37,43 & 38,68 & 38,68 & 37,43 & 37,43 \\
Paleta & 37,45 & 37,43 & 38,68 & 38,68 & 37,43 & 37,43 \\
Gelo & 15 & 15 & 15 & 15 & 15 & 15 \\
Óleo de linhaça dourada & 0 & 0 & 2,5 & 2,5 & 5 & 5 \\
Gordura vegetal & 5 & 5 & 0 & 0 & 0 & 0 \\
Eritorbato de sódio & 0 & 0,05 & 0 & 0 & 0 & 0 \\
Extrato de alecrim & 0 & 0 & 0,05 & 0 & 0,05 & 0 \\
Extrato de chá verde & 0 & 0 & 0 & 0,05 & 0 & 0,05 \\
\hline
\end{tabular}

\subsection{PROCESSAMENTO DOS HAMBÚRGUERES BOVINOS}

O processamento dos hambúrgueres ocorreu na planta de processamento de carnes da Faculdade de Engenharia de Alimentos da Universidade Estadual de Campinas (UNICAMP). Inicialmente, os pedaços de carne a $4^{\circ} \mathrm{C}$ foram moídos em moedor de carnes (C.A.F.®, Brasil), com disco de $3,5 \mathrm{~mm}$. Na misturadeira (Super Cutter Sire $®$, Brasil) foram adicionados os ingredientes na seguinte ordem: as carnes com metade do gelo, condimentos (alho e cebola em pó), extratos naturais ou eritorbato de sódio, o restante do gelo, sal, maltodextrina e gordura vegetal ou óleo de linhaça. Após a homogeneização de cada formulação, essas foram acondicionadas em sacos plásticos de polietileno de baixa densidade (PEBD) e armazenadas em freezer com temperatura de $0^{\circ} \mathrm{C} \mathrm{a}-1^{\circ} \mathrm{C}$ por, aproximadamente, 1 hora para facilitar a moldagem. Em seguida, a massa foi moldada em hamburgueira manual (Muller ${ }^{\circledR}$, Brasil), contendo cerca de $110 \mathrm{~g}$ e os hambúrgueres formados foram levados a câmara de congelamento. As amostras congeladas foram embaladas em sacos plásticos de PEBD, identificadas e armazenadas em freezer $\left(-18^{\circ} \mathrm{C}\right)$ durante o período do estudo.

\subsection{AVALIAÇÃO DA ESTABILIDADE À OXIDAÇÃO LIPÍDICA}

Nas seis formulações, foram realizados três testes para se avaliar a oxidação lipídica, dois dias após o processamento: do produto cru, cozido e utilizando teste acelerado para se verificar a incidência de warmed-over flavour (WOF). O teste acelerado de WOF consistiu em expor os 
hambúrgueres cozidos à temperatura ambiente por 1 hora, e depois sob refrigeração a $5{ }^{\circ} \mathrm{C}$ por 1 hora e 45 minutos. As amostras foram homogeneizadas, misturadas a uma solução de BHA (butilato de hidroxianisol) e congeladas em freezer à temperatura de $-18^{\circ} \mathrm{C}$. Posteriormente, foram analisados os teores de substâncias reativas ao ácido tiobarbitúrico (TBARS). Os resultados são expressos em unidades de absorbância por unidade de massa de amostras ou em "valor de TBARS" ou "número de TBARS", definidos como a massa, em mg, de malonaldeído por $\mathrm{Kg}$ (MDA Kg) de amostra (TARLADGIS et al., 1960).

\subsection{COCÇÃO DOS HAMBÚRGUERES BOVINOS}

Para a realização das análises sensoriais, determinação de perdas por cocção, percentual de encolhimento e teste de oxidação acelerada warmed-over flavour os hambúrgueres congelados foram grelhados em chapa elétrica com grelha nos lados superior e inferior (George Foreman, tamanho jumbo, Lean Mean Fat reducing Grilling Machine ${ }^{\circledR}$, USA), aquecida a $200^{\circ} \mathrm{C}$. Controlouse a temperatura interna do hambúrguer usando temômetro digital B 345 (Micronal@, Brasil) com termopar acoplado até que atingisse $72^{\circ} \mathrm{C}$ no centro. $\mathrm{O}$ tempo de fritura durou, em média, 7 a 8 minutos.

\subsection{ANÁLISES FÍSICO-QUÍMICAS}

A determinação do teor de gordura foi realizada de acordo com a metodologia descrita por Bligh e Dyer (1959). Todas as determinações foram realizadas em triplicata $24 \mathrm{~h}$ após o processamento.

As medições de cor foram feitas usando um colorímetro Hunter Lab (Colourquest- II Hunter Associates Laboratory Inc., Virginia, EUA) calibrado previamente, operando com iluminante D65, 20-mm de tamanho de porta, ângulo do observador $10^{\circ}$, no modo RSEX e no sistema de cor CIELAB, onde $L^{*}$ representa a luminosidade, $a^{*}$, o eixo vermelho-verde e $b^{*}$, o eixo amarelo-azul. As variáveis de cor foram medidas em quatro pontos das amostras de hambúrgueres crus. Os ensaios foram realizados em triplicata para cada tratamento. As amostras foram avaliadas $24 \mathrm{~h}$ após o processamento.

$\mathrm{O} \mathrm{pH}$ foi determinado pela medição direta usando um medidor de pH (MA 120 Mettler Toledo Industria e Comercio Ltda, SP, Brasil) nas amostras de hambúrgures crus 24h após o processamento. A atividade de água (Aw) foi medida por um aparelho de atividade de água modelo Aqualab Cx 2T (Decagon Devices Inc., Pullman, EUA). Três hambúrgueres por tratamento foram utilizados para avaliar o $\mathrm{pH}$ e a Aw e cada análise foi realizada em triplicata.

O percentual de perdas na cocção foi calculado pela diferença entre o peso da amostra crua e da cozida, de acordo com Hur, Jin e Kim (2008), expresso em percentual de perdas por cocção: (peso da amostra crua- peso da amostra cozida)/ (peso da amostra crua) x 100. O percentual de encolhimento foi determinado segundo Berry (1992), através da seguinte relação: (diâmetro da amostra crua - diâmetro da amostra cozida) / diâmetro da amostra crua x 100.

\subsection{ANÁLISE SENSORIAL}

A análise sensorial, devidamente aprovada pelo Comitê de Ética em Pesquisa da Universidade Estadual de Campinas, SP, Brasil (Parecer do Projeto CEP No 273/2009) foi realizada através de um teste hedônico para avaliar o grau de aceitação dos produtos elaborados, em relação aos atributos de cor, aroma, sabor, textura e aparência. A análise sensorial foi realizada por 50 participantes recrutados entre estudantes, funcionários e professores no campus. As amostras foram servidas em blocos completos balanceados, de forma monádica, em pratos plásticos codificados com algarismos de três dígitos, em cabines individuais. Utilizou-se escala hedônica mista estruturada de 
9 pontos, com extremos que variaram de "desgostei muitíssimo a "gostei muitíssimo" (MEILGAARD, CARR e CIVLLE, 2006).

O teste de aceitação global e intenção de compra foi aplicado mediante dois pontos específicos entre gostei e não gostei e compraria e não compraria, respectivamente (MOSKOWITZ, 1994), aplicado também a 50 julgadores.

\subsection{ANÁLISE ESTATÍSTICA}

Os dados foram avaliados através de análise de variância (ANOVA). As médias foram comparadas pelo teste de Tukey a um nível de confiança de $5 \%(p<0,05)$, utilizando o programa estatístico SAS, versão 9.1 (SAS, 2002).

\section{RESULTADOS E DISCUSSÃO}

\subsection{AVALIAÇÃO DA ESTABILIDADE À OXIDAÇÃO LIPÍDICA}

A análise de TBARS foi utilizada para avaliar o índice de oxidação dos hambúrgueres com perfil lipídico modificado e com antioxidantes nas amostras cruas, cozidas e submetidas ao teste de incidência de warmed-over flavour (WOF) (Tabela 2).

\section{TABELA 2- VALORES DE TBARS DOS HAMBÚRGUERES CRUS, COZIDOS E APÓS TESTE DE INCIDÊNCIA DE WARMED-OVER FLAVOUR (WOF)}

\begin{tabular}{cccc}
\hline Tratamentos & Hambúrguer Cru & Hambúrguer Cozido & Hambúrguer WOF \\
\hline FP & $0,071^{\mathrm{d}} \pm 0,001$ & $0,383^{\mathrm{d}} \pm 0,020$ & $0,729^{\mathrm{c}, \mathrm{d}} \pm 0,017$ \\
FE & $0,059^{\mathrm{d}} \pm 0,001$ & $0,335^{\mathrm{d}} \pm 0,014$ & $0,611^{\mathrm{d}} \pm 0,018$ \\
F1 & $0,144^{\mathrm{b}} \pm 0,006$ & $0,722^{\mathrm{a}} \pm 0,007$ & $0,822^{\mathrm{c}, \mathrm{d}} \pm 0,014$ \\
F2 & $0,077^{\mathrm{d}} \pm 0,007$ & $0,496^{\mathrm{c}} \pm 0,009$ & $0,870^{\mathrm{c}} \pm 0,016$ \\
F3 & $0,201^{\mathrm{a}} \pm 0,002$ & $0,562^{\mathrm{b}, \mathrm{c}} \pm 0,019$ & $1,652^{\mathrm{a}} \pm 0,080$ \\
F4 & $0,110^{\mathrm{c}} \pm 0,006$ & $0,608^{\mathrm{b}} \pm 0,018$ & $1,102^{\mathrm{b}} \pm 0,071$ \\
\hline
\end{tabular}

\footnotetext{
* Valores representam a media \pm desvio padrão; Valor TBARS: mg de malonaldeido (MDA)/Kg de amostra; a,b,c,d Médias na mesma coluna com letras iguais não diferem significativamente $\mathrm{p}<0,05$ (Teste de Tukey). FP: Controle ( $5 \%$ de gordura vegetal, sem antioxidantes); FE: $5 \%$ de gordura vegetal e $0,05 \%$ de eritorbato de sódio; F1: $2,5 \%$ de OL e $0,05 \%$ de EA; F2: $2,5 \%$ de OL e $0,05 \%$ de ECV; F3: $5 \%$ de OL e $0,05 \%$ de EA; F4: $5 \%$ de OL e $0,05 \%$ de ECV. OL: óleo de linhaça; EA: extrato de alecrim; ECV: extrato de chá verde.
}

A formação de TBARS nas amostras cruas variou de 0,059 em FE a 0,201 mg de MDA/ $\mathrm{Kg}$ de amostra em F3, que diferiu significativamente $(p>0,05)$ de todos os outros tratamentos. Entre as formulações FE, FP e F2 não houve diferenças significativas $(p<0,05)$, o que demonstra efetiva proteção antioxidante do extrato de chá verde na amostra crua com até $2,5 \%$ de óleo de linhaça em sua composição. Já com teor maior de óleo de linhaça (5\%) a proteção antioxidante foi menos eficaz, sendo que na amostra com extrato de chá verde (F4) o índice de TBARS encontrado foi significativamente menor $(p>0,05)$ quando comparada à amostra com extrato de alecrim $(F 3)$, contudo estes valores foram acima dos encontrados para os tratamentos FE, FP e F2. Valência et al. (2008) estudaram a influencia da adição de $0,02 \%$ de catequinas de chá verde em salsichas com $15 \%$ de gordura saturada substituída por óleo de linhaça, encontrando valores de TBA de 0,330 mg de MDA/ $\mathrm{Kg}$ de massa no produto cru que não diferiu $(p<0,05)$ de sua amostra controle com gordura suína. 
Nos hambúrgueres cozidos os níveis de oxidação lipídica foram maiores do que nas amostras cruas devida à natureza pró-oxidante do processo de cozimento. A formação de TBARS nas amostras cozidas variou de $0,335 \mathrm{em} F E$, que não diferiu significativamente $(p<0,05)$ de $F P$, a $0,722 \mathrm{mg}$ de MDA/ Kg de amostra em F1, que diferiu significativamente $(p>0,05)$ de todos os outros tratamentos. Entre as amostras cozidas com $5 \%$ de óleo de linhaça (F3 e F4) não houve diferenças significativas $(p<0,05)$, o que demonstra atuação semelhante dos antioxidantes naturais quando submetidos ao tratamento térmico com mesmo perfil lipídico.

No teste acelerado para se verificar a incidência de warmed-over flavour (WOF), foram encontrados valores de TBARS entre 0,611 em FE, que não diferiu significativamente $(p<0,05)$ de FP e F1, a 1,652 mg de MDA/Kg de amostra em F3, que diferiu significativamente $(p>0,05)$ de todos os outros tratamentos. A percepção sensorial da rancidez oxidativa em produtos cárneos é sugerida por Gray e Pearson (1987) como sendo de $1 \mathrm{mg}$ de MDA/Kg de amostra. Das amostras submetidas ao tratamento de WOF, as formulações F3 e F4, apresentaram valores acima do limiar perceptível segundo os autores citados, o que indica que estas formulações podem não ser recomendadas para linhas fast food e produtos prontos para consumo. Nos tratamentos com $2,5 \%$ de óleo de linhaça submetidos ao teste de incidência de WOF houve proteção antioxidante similar entre os extratos de alecrim e chá verde onde F1 não diferiu significativamente $(p<0,05)$ de $F 2$.

\subsection{ANÁLISES FÍSICO-QUÍMICAS}

A Tabela 3 demonstra os resultados obtidos nas análises físico-químicas de determinação de teor de lipídios, aw, pH, perdas por cocção e \% de encolhimento dos hambúrgueres com óleo de linhaça, gordura vegetal e antioxidantes. Os teores de lipídeos das amostras FP, FE e F4, com maior quantidade de gordura adicionada $(5 \%)$ foram significativamente iguais entre si $(p<0,05)$ diferindo $(p>0,05)$ apenas de F3, também com $5 \%$ de gordura acrescida. Possivelmente, esta diferença se deve às perdas de óleo de linhaça durante o processamento ou uma porção muscular com menor teor de gordura intramuscular.

\section{TABELA 3- RESULTADOS DAS ANÁLISES FÍSICO-QUÍMICAS DOS HAMBÚRGUERES BOVINOS ADICIONADOS DE ÓLEO DE LINHAÇA DOURADA, GORDURA VEGETAL E ANTIOXIDANTES}

\begin{tabular}{cccccc}
\hline Tratamentos & Lipídios (\%) & $\begin{array}{c}\text { Atividade de } \\
\text { água (Aw) }\end{array}$ & $\mathbf{p H}$ & $\begin{array}{c}\text { Perdas por } \\
\text { cocção (\%) }\end{array}$ & Encolhimento (\%) \\
\hline FP & $5,4^{\mathrm{a}} \pm 0,19$ & $0,98^{\mathrm{a}} \pm 0,004$ & $5,68^{\mathrm{c}} \pm 0,02$ & $33,34^{\mathrm{a}} \pm 2,51$ & $23,74^{\mathrm{a}} \pm 1,02$ \\
FE & $5,41^{\mathrm{a}} \pm 0,18$ & $0,98^{\mathrm{a}} \pm 0,01$ & $5,54^{\mathrm{d}} \pm 0,04$ & $34,77^{\mathrm{a}} \pm 0,99$ & $19,73^{\mathrm{a}} \pm 3,21$ \\
F1 & $3,2^{\mathrm{c}} \pm 0,04$ & $0,97^{\mathrm{a}} \pm 0,004$ & $5,86^{\mathrm{a}} \pm 0,04$ & $38,12^{\mathrm{a}} \pm 1,13$ & $20,49^{\mathrm{a}} \pm 1,08$ \\
F2 & $3,57^{\mathrm{c}} \pm 0,06$ & $0,98^{\mathrm{a}} \pm 0,004$ & $5,69^{\mathrm{b}, \mathrm{c}} \pm 0,04$ & $33,02^{\mathrm{a}} \pm 3,13$ & $21,54^{\mathrm{a}} \pm 2,06$ \\
F3 & $4,76^{\mathrm{b}} \pm 0,15$ & $0,99^{\mathrm{a}} \pm 0,004$ & $5,75^{\mathrm{b}, \mathrm{c}} \pm 0,03$ & $32,84^{\mathrm{a}} \pm 1,40$ & $19,87^{\mathrm{a}} \pm 0,11$ \\
\hline
\end{tabular}

* Valores representam a media \pm desvio padrão;

a,b,c,d Médias na mesma coluna com letras iguais não diferem significativamente $p<0,05$ (Teste de Tukey). FP: Controle (5\% de gordura vegetal, sem antioxidantes); FE: $5 \%$ de gordura vegetal e 0,05\% de eritorbato de sódio; F1: $2,5 \%$ de OL e $0,05 \%$ de EA; F2: 2,5\% de OL e 0,05\% de ECV; F3: $5 \%$ de OL e 0,05\% de EA; F4: 5\% de OL e 0,05\% de ECV. OL: óleo de linhaça; EA: extrato de alecrim; ECV: extrato de chá verde. 
A RDC n 54, de 12 de novembro de 2012 estabelece requisitos para regulamentação da informação nutricional complementar contida nos rótulos. De acordo com as informações contidas nesta RDC pôde-se verificar que os hambúrgueres elaborados neste estudo possuem, de modo geral, reduzido conteúdo de gordura. Para utilização de informação nutricional complementar é necessário uma avaliação comparativa com o valor médio do conteúdo de três alimentos de referência comercializados no país de processamento, neste caso o Brasil. Utilizando-se de valores médios de gorduras totais de hambúrguer bovino de marcas comercializadas no país, têm-se em média $11 \mathrm{~g}$ de gorduras totais por porção de hambúrguer ( $80 \mathrm{~g}$, equivalente a 1 unidade e $1 / 2$ ). Os hambúrgueres elaborados neste estudo, considerando o maior percentual de gordura acrescida (5\%), possuíam em média $4,23 \mathrm{~g}$ de gorduras totais por porção, portanto, obtiveram uma redução significativa de $61,5 \%$ de gorduras totais, além de possuir um perfil lipídico mais saudável, rico em ácidos graxos polinsaturados nos tratamentos com adição de óleo de linhaça dourada. Ainda segundo a RDC a redução mínima deve ser de $25 \%$ do conteúdo de gorduras totais para que se adote o termo ou expressão "reduzido em" (BRASIL, 2012).

Não houve diferenças significativas $(p<0,05)$ nos valores de atividade de água e perdas por cocção em todas as formulações. Hur, Jin e Kim (2008) investigaram os efeitos da substituição da gordura suína por azeite de oliva $(5 \%)$ e ingredientes extensores (proteína isolada de soja, carragena e maltodextrina) em produtos cárneos reestruturados de carne suína e também não encontraram diferenças significativas $(p<0,05)$ nas perdas por cocção entre as amostras de produtos cárneos.

A habilidade da carne fresca e produtos cárneos em reter sua umidade durante processos como corte, moagem e cozimento, conceito de capacidade de retenção de água (CRA) (PEARCE et al., 2011), é indiscutivelmente uma das propriedades mais importantes de qualidade porque afeta tanto aspectos quantitativos quanto qualitativos (HUFF-LONERGAN e LONERGAN, 2005). A CRA também é influenciada por fatores como o $\mathrm{pH}$, que por sua vez, está fortemente relacionado com as perdas por cocção em produtos cárneos (HUGUES et al., 2014). No presente estudo, os valores de $\mathrm{pH}$ encontrados situaram-se entre 5,54 a 5,86 , semelhante a faixa de valores também encontradas por Hur, Jin e Kim (2008) que se situou entre 5,75 a 5,82. Houve diferença significativa $(p>0,05)$ entre as formulações F1 e FE, porém, esta diferença deve-se ao baixo desvio padrão entre as repetições dos ensaios. Todas as formulações encontram-se dentro da faixa de valores de $\mathrm{pH}$ normalmente encontrados para produtos cárneos processados.

Em relação ao percentual de encolhimento também não houve diferenças significativas $(p<0,05)$ entre todas as formulações. Serdaroglu e Degirmencioglu (2004) relataram que o nível de gordura afetou o percentual de encolhimento de suas amostras de bolos de carne (típicos da Turquia), quando o nível de gordura diminuiu de $20 \%$ para $5 \%$, o valor de encolhimento foi significativamente menor $(p>0,05)$. De modo geral, produtos cárneos reestruturados tendem a encolher durante 0 processo de cozimento, devido à desnaturação das proteínas da carne com a consequente perda de água, além disso, a perda de gordura também pode contribuir para o processo de encolhimento. No presente estudo os valores de \% de encolhimento situaram-se entre 19,73 a 23,74, semelhante à faixa de valores encontrada por Serdaroglu e Degirmencioglu (2004) que se situou entre 19,3 a 25,3.

Os resultados da determinação de cor dos hambúrgueres com diferentes níveis de adição de óleo de linhaça, gordura vegetal e antioxidantes são apresentados na Tabela 4. 


\section{TABELA 4- AVALIAÇÃO OBJETIVA DA COR DOS HAMBÚRGUERES BOVINOS ADICIONADOS DE ÓLEO DE LINHAÇA DOURADA, GORDURA VEGETAL E ANTIOXIDANTES}

\begin{tabular}{cccc}
\hline Tratamentos & $\mathbf{L}^{*}$ & $\mathbf{a}^{*}$ & $\mathbf{b}^{*}$ \\
\hline FP & $50,31^{\mathrm{a}, \mathrm{b}} \pm 1,69$ & $15,39^{\mathrm{a}} \pm 1,99$ & $18,08^{\mathrm{b}, \mathrm{c}} \pm 1,62$ \\
FE & $51,20^{\mathrm{a}} \pm 1,89$ & $9,8^{\mathrm{b}} \pm 0,90$ & $16,82^{\mathrm{c}} \pm 1,27$ \\
F1 & $48,73^{\mathrm{b}} \pm 1,56$ & $13,72^{\mathrm{a}} \pm 0,80$ & $18,81^{\mathrm{a}, \mathrm{b}} \pm 1,00$ \\
F2 & $48,94^{\mathrm{a}, \mathrm{b}} \pm 1,14$ & $14,24^{\mathrm{a}} \pm 0,96$ & $19,71^{\mathrm{a}, \mathrm{b}} \pm 1,16$ \\
F3 & $48,91^{\mathrm{a}, \mathrm{b}} \pm 1,12$ & $14,84^{\mathrm{a}} \pm 0,95$ & $20,39^{\mathrm{a}} \pm 1,16$ \\
F4 & $48,19^{\mathrm{b}} \pm 0,69$ & $14,96^{\mathrm{a}} \pm 0,51$ & $20,14^{\mathrm{a}} \pm 0,53$ \\
\hline
\end{tabular}

a,b,c Médias na mesma coluna com letras iguais não diferem significativamente $p<0,05$ (Teste de Tukey). FP: Controle $(5 \%$ de gordura vegetal, sem antioxidantes); FE: $5 \%$ de gordura vegetal e $0,05 \%$ de eritorbato de sódio; F1: 2,5\% de OL e 0,05\% de EA; F2: $2,5 \%$ de OL e 0,05\% de ECV; F3: $5 \%$ de OL e 0,05\% de EA; F4: $5 \%$ de OL e 0,05\% de ECV. OL: óleo de linhaça; EA: extrato de alecrim; ECV: extrato de chá verde.

Os valores de $L^{*}$ que representam a luminosidade foram semelhantes entre os tratamentos e não houve diferenças significativas $(p<0,05)$ entre a formulação controle FP $(5 \%$ de gordura vegetal) e F1, F2, F3 e F4. Tais resultados demonstram que os níveis de substituição da gordura vegetal pelo óleo de linhaça selecionados para este estudo não impactaram neste parâmetro $\left(L^{*}\right)$ de avaliação de cor objetiva. Em outros níveis de substituição, como por exemplo, o nível avaliado por Valência et al. (2008) em seu estudo, onde 15\% da gordura suína foi substituída por óleo de linhaça em salsichas, foram encontrados valores de $L^{*}$ significativamente maiores $(p>0,05)$.

Em relação à intensidade da cor vermelha $\left(a^{*}\right)$ a formulação FE diferiu significativamente $(p>0,05)$ das demais. Uma hipótese possível para este resultado em FE se deve a presença do eritorbato de sódio, um agente redutor, que manteve os pigmentos heme no estado ferroso com maior predominância do pigmento purpúreo desoximioglobina o que explicaria o menor valor de $a^{*}$ observado. Tais dados corroboram com estudos de Suman et al. (2005) que avaliaram o efeito do eritorbato de sódio em bolos de carne sob diferentes condições de estocagem e também encontraram menores valores de $a^{*}$ em suas amostras com este antioxidante submetidas ao congelamento.

Em relação ao parâmetro $b^{*}$, que representa o eixo amarelo-azul e indica a intensidade de cor amarelo nos produtos, foi observado um aumento nos valores para os tratamentos com maiores níveis de óleo de linhaça, contudo não houve diferenças significativas $(p<0,05)$ entre F1, F2 e amostra controle FP. Estes resultados demonstram que níveis de adição de até 2,5\% de óleo de linhaça nos hambúrgueres não influeciaram o valor de $b^{*}$. Já os tratamentos F3 e F4 com $5 \%$ de óleo de linhaça diferiram significativamente $(p>0,05)$ de FP e FE. Estudos conduzidos por Choi et al. (2009) também mostraram valores maiores de $b^{*}$ nas formulações de produtos cárneos emulsionados com óleo de linhaça e outros óleos vegetais.

\subsection{ANÁLISE SENSORIAL}

A avalição sensorial realizada para as amostras de hambúrguer nos diferentes tratamentos indicou que a substituição da gordura vegetal pelo óleo de linhaça e adição de antioxidantes influenciou alguns atributos sensoriais dos produtos (Tabela 5). 
TABELA 5- AVALIAÇÃO SENSORIAL DOS HAMBÚRGUERES BOVINOS

\begin{tabular}{cccccc}
\hline Tratamentos & Aparência & Aroma & Cor & Sabor & Textura \\
\hline FP & $6,52^{\mathrm{a}} \pm 1,31$ & $6,70^{\mathrm{a}} \pm 1,31$ & $6,62^{\mathrm{a}, \mathrm{b}} \pm 1,26$ & $7,06^{\mathrm{a}} \pm 1,22$ & $6,10^{\mathrm{a}, \mathrm{b}} \pm 1,69$ \\
FE & $5,66^{\mathrm{a}} \pm 1,87$ & $6,06^{\mathrm{a}} \pm 1,53$ & $5,78^{\mathrm{b}} \pm 1,90$ & $6,14^{\mathrm{b}, \mathrm{c}} \pm 1,82$ & $6,38^{\mathrm{a}, \mathrm{b}} \pm 1,74$ \\
F1 & $6,22^{\mathrm{a}} \pm 1,56$ & $6,32^{\mathrm{a}} \pm 1,22$ & $6,32^{\mathrm{a}, \mathrm{b}} \pm 1,54$ & $5,70^{\mathrm{c}} \pm 1,68$ & $5,60^{\mathrm{b}} \pm 1,63$ \\
F2 & $6,22^{\mathrm{a}} \pm 1,52$ & $6,32^{\mathrm{a}} \pm 1,35$ & $6,16^{\mathrm{a}, \mathrm{b}} \pm 1,64$ & $6,92^{\mathrm{a}, \mathrm{b}} \pm 1,40$ & $6,90^{\mathrm{a}} \pm 1,47$ \\
F3 & $6,44^{\mathrm{a}} \pm 1,66$ & $6,08^{\mathrm{a}} \pm 1,47$ & $6,68^{\mathrm{a}} \pm 1,45$ & $5,58^{\mathrm{c}} \pm 1,64$ & $6,14^{\mathrm{a}, \mathrm{b}} \pm 1,73$ \\
F4 & $6,56^{\mathrm{a}} \pm 1,59$ & $6,42^{\mathrm{a}} \pm 1,51$ & $6,64^{\mathrm{a}, \mathrm{b}} \pm 1,51$ & $6,00^{\mathrm{c}} \pm 1,77$ & $6,52^{\mathrm{a}, \mathrm{b}} \pm 1,63$ \\
\hline
\end{tabular}

* Valores representam a media \pm desvio padrão;

a,b,c Médias na mesma coluna com letras iguais não diferem significativamente $p<0,05$ (Teste de Tukey). FP: Controle (5\% de gordura vegetal, sem antioxidantes); FE: $5 \%$ de gordura vegetal e 0,05\% de eritorbato de sódio; F1: 2,5\% de OL e 0,05\% de EA; F2: 2,5\% de OL e 0,05\% de ECV; F3: $5 \%$ de OL e 0,05\% de EA; F4: $5 \%$ de OL e 0,05\% de ECV. OL: óleo de linhaça; EA: extrato de alecrim; ECV: extrato de chá verde.

Os provadores identificaram diferenças de sabor da amostra controle em relação às demais, atribuindo nota maior à formulação FP que diferiu significativamente $(p>0,05)$ das demais, com exceção de $F 2$. Não houve diferenças significativas $(p<0,05)$ para este atributo entre os tratamentos FE, F1, F2, F3 e F4, o que demonstra que os antioxidantes presentes e níveis de gordura e óleo influenciaram de modo semelhante o atributo sabor na percepção dos consumidores. Estudo conduzido por Salcedo-Sandoval et al. (2015) demonstrou que níveis maiores de adição de misturas de óleos saudáveis (azeite de oliva, linhaça e peixe) que substituíram 100\% da gordura suína em produtos cárneos reestruturados de carne suína afetaram atributos de sabor e aceitação global.

$O$ atributo sensorial cor obteve notas semelhantes entre quase todos os tratamentos, não sendo encontradas diferenças significativas $(p<0,05)$ entre as formulações FP, F1, F2, F3 e F4. A formulação FE diferiu significativamente $(p>0,05)$ somente de $F 3$. A avaliação objetiva de cor também identificou diferenças entre FE e as demais corroborando com a análise sensorial.

Os atributos sensoriais aroma e aparência foram avaliados pelos consumidores de forma semelhante e diferenças significativas não foram encontradas $(p<0,05)$ entre todos os tratamentos. Em relação a textura, diferenças significativas $(p>0,05)$ foram encontradas somente entre $F 1$ e $F 2$, contudo, de modo geral, as notas também foram muito semelhantes para este atributo. Novello e Pollonio (2012) avaliaram níveis de 0 a 10\% de adição de óleo, farinha e semente de linhaça dourada em hambúrguer bovino e concluíram que níveis de adição de até $5 \%$ de cada ingrediente alcançou boa aceitação sensorial, e também não encontraram diferenças significativas $(p<0,05)$ entre os tratamentos para os atributos aroma, aparência e textura.

A Figura 1 apresenta o histograma de intenção de compra e aceitação global das formulações submetidas à avaliação sensorial. 


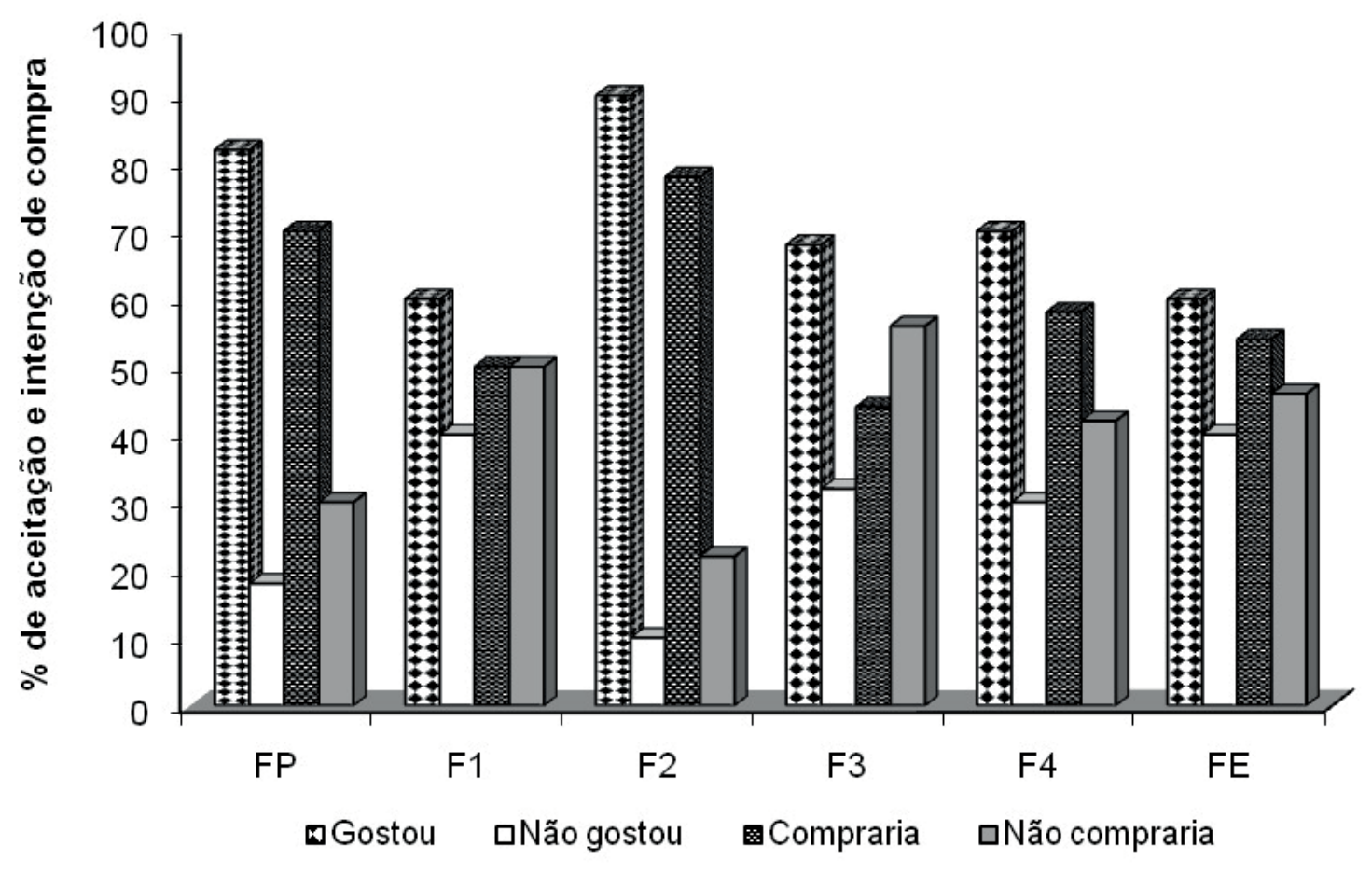

\section{FIGURA 1 - HISTOGRAMA DA ACEITAÇÃO GLOBAL E INTENÇÃO DE COMPRA DOS HAMBÚRGUERES BOVINOS}

FP: Controle ( $5 \%$ de gordura vegetal, sem antioxidantes); FE: $5 \%$ de gordura vegetal e $0,05 \%$ de eritorbato de sódio; F1: $2,5 \%$ de OL e $0,05 \%$ de EA; F2: $2,5 \%$ de OL e $0,05 \%$ de ECV; F3: $5 \%$ de OL e 0,05\% de EA; F4: $5 \%$ de OL e 0,05\% de ECV. OL: óleo de linhaça; EA: extrato de alecrim; ECV: extrato de chá verde.

Em relação ao percentual de aceitação global e intenção de compra, pôde-se perceber que a formulação $\mathrm{F} 2$, adicionada de $2,5 \%$ de óleo de linhaça e $0,05 \%$ de extrato de chá verde apresentou maior aceitação (90\%) e melhor intenção de compra (78\%). Estes resultados podem justificar as melhores notas obtidas para os atributos de sabor e textura. A menor aceitação global foi atribuída para as formulações F1 e FE, ambas com 40 \% de rejeição. Novello e Pollonio (2012) em seu estudo com vários níveis de adição de farinha, óleo e semente de linhaça dourada em hambúrgueres bovinos concluíram que os índices de rejeição mais elevados em relação à aceitação global e intenção de compra foram nos produtos com níveis acima de $5 \%$ dos produtos à base de linhaça dourada.

\section{CONCLUSÃO}

A estratégia de reformulação descrita, com base na substituição da gordura vegetal por óleo de linhaça dourada e antioxidantes naturais em hambúrguer bovino demonstrou ser possível o desenvolvimento de produtos mais saudáveis com características tecnológicas adequadas e satisfatória aceitação sensorial. Os valores de gorduras totais foram consideravelmente reduzidos (redução de 61,5\%) e o perfil lipídico foi melhorado com o acréscimo de ácidos graxos polinsaturados. Isto significa que pela legislação brasileira os hambúrgueres reformulados poderiam se beneficiar com informação nutricional complementar, como por exemplo, a expressão "reduzido em gorduras totais". Os produtos reformulados também tiveram satisfatórias características tecnológicas (perdas 
por cocção e encolhimento), contudo, em níveis de $5 \%$ de adição de óleo de linhaça houve ligeiras modificações no valor de $b^{*}$, mas não perceptíveis pelos consumidores conforme demonstrou a avaliação sensorial. Além disso, em níveis de adição de até $2,5 \%$ de óleo de linhaça houve eficácia dos antioxidantes naturais na proteção contra oxidação lipídica, porém com desempenho inferior ao antioxidante sintético eritorbato de sódio, o que demonstra que novos estudos com concentrações mais elevados dos extratos naturais devem ser realizados a fim de se determinar o nível ideal para proteção antioxidante próxima a exercida pelo composto sintético.

\section{ABSTRACT \\ EFFECT OF ADDED ROSEMARY EXTRACT, GREEN TEA AND LINSEED OIL ON OXIDATIVE STABILITY AND PHYSICOCHEMICAL AND SENSORY PROPERTIES OF BOVINE HAMBURGER}

The effects of the addition of rosemary extract and green tea and substitution of vegetable fat by golden linseed oil on the physicochemical properties and sensory in bovine hamburgers were studied. The TBARS method was used to evaluate the oxidative stability and acceptance testing in sensory evaluation, in addition to performing physicochemical analysis like objective determination of color, lipids, $\mathrm{pH}$, water activity, cooking losses and shrinkage. The addition of linseed oil had an influence the increase in yellow color intensity at levels above $2.5 \%$. The sensory evaluation showed better acceptance of F2, by sensory panel. There were no significant difference $(p<0.05)$ between the appearance and aroma of products. The antioxidants and levels of fat and oil influenced similarly the attribute flavor in the perception of consumers. When the antioxidant activity of plant extracts by TBARS values was evaluated, it was found better performance of sodium erythorbate in relation to the plant extracts. Among the plant extracts was found that, for the concentration of $2.5 \%$ linseed oil, both showed similar antioxidant activity. The values of total fat were significantly reduced (up to $61.5 \%$ ) and the lipid profile was improved with the addition of polyunsaturated fatty acids. The strategy described formulation, based on the substitution of vegetable fat by golden linseed oil and natural antioxidants in hamburger proved to be technologically feasible and an alternative consumption of healthier meat products.

KEY-WORDS: HAMBURGER; LINSEED OIL; LIPID OXIDATION; GREEN TEA; ROSEMARY.

\section{REFERÊNCIAS}

1 ALMAJANO, M.P.; CARBO, R.; JIMENEZ, J.A.L.; GORDON, M.H. Antioxidant and antimicrobial activities of tea infusions. Food Chemistry, v.108, p.55-63, 2008.

2 ANSORENA, D.; ASTIASARÁN, I. Effect of storage and packaging on fatty acid composition and oxidation in dry fermented sausages made with added olive oil and antioxidants. Meat Science, v. 67, p. 237-244, 2004.

3 BERRY, B. W. Low fat level effects on sensory, shear, cooking, and chemical properties of ground beef patties. Journal of Food Science. v. 57, n.3, p. 537-540, 1992.

4 BLIGH, E.G.; DYER, W.J. A rapid method of total lipid extraction and purification. Canadian Journal of Biochemistry and Physiology, v.37, n.8, p.911-917, 1959.

5 BOZKURT, H. Utilization of natural antioxidants: Green tea extract and Thymbra spicata oil in Turkish dry-fermented sausage. Meat Science, v.73, p.442-450, 2006.

6 BRASIL. Ministério da Saúde. Agência Nacional de Vigilância Sanitária. RDC n 54, de 12 de novembro de 2012. Aprova o regulamento técnico sobre informação nutricional complementar. Diário oficial [da] República Federativa do Brasil, Brasília, 13 de novembro de 2012. Seção 1, p.122. 
7 BYRNE, D.V.; BREDIE, W.L.P. ; BAK, L.S. ; BERTELSEN, G.; MARTENS, H; MARTENS. Sensory and chemical analysis of cooked porcine meat patties in relation to warmed-over flavour and pre-slaughter stress. Meat Science, v.59, p.229249, 2001

8 BYRNE, D.V.; O'SULLIVAN, M.G.; BREDIE, W.L.P.; ANDERSEN, H.J.; MARTENS, M. Descriptive sensory profiling and physical/chemical analyses of warmed-over flavour in pork patties from carriers and non-carriers of the RN_allele. Meat Science, v. 63, p. 211-224, 2003.

9 CHOI, Y. S.; CHOI, J. H.; HAN, D. J.; KIM, H. Y.; LEE, M. A.; KIM, H. W.; JEONG, J. Y.; KIM, C. J. Characteristics of low-fat meat emulsion systems with pork fat replaced by vegetable oils and rice bran fiber. Meat Science, v.82, n.2, p. 266-271, 2009.

10 FERNANDEZ-LOPEZ, J.; ZHI, N.; ALESON-CARBONELL, L.; PEREZ-ALVAREZ, J.A.; KURI, V. Antioxidant and antibacterial activities of natural extracts: application in beef meatballs. Meat Science, v.69, p. 371-380, 2005.

11 GEORGANTELIS, D.; AMBROSIADIS, I.; KATIKOU, P.; BLEKAS, G.; GEORGAKIS, S.A. Effect of rosemary extract, chitosan and a-tocopherol on microbiological parameters and lipid oxidation of fresh pork sausages stored at $4{ }^{\circ} \mathrm{C}$. Meat Science, v.76, p. 172-181, 2007.

12 GIESE, J. Developing low-fat meat products. Food Technology, v.46, p.100-108, 1992.

13 GRAY, J.I.; PEARSON, A.M. Rancidity and warmed-over flavor. In: A.M.PEARSON, A.M. e DUTSON, T.R. (Eds). Restructured Meat and Poultry Products, Advances in Meat Research. New York: Van Nostrand Reinhold Company, 1987. Chap. 6, p.221-269.

14 GRAY, J.I.; GOMAA, E.A.; BUCKLEY, D.J. Oxidative quality and shelf life of meats. Meat Science, v.43, $n^{\circ}$ suppl 1, p. 111-123, 1996.

15 GRÜN, I.U.; AHN, J.; CLARKE, A. D.; LORENZEN, C.L. Reducing oxidation of meat. Food Technology, v.1, p. 36-43, 2006.

16 HORZIC, D.; KOMES, D.; GANIC, K.K.; IVEKOVIC, D.; KARLOVIC, D. The composition of polyphenols and methylxanthines in teas and herbal infusions. Food Chemistry, v. 115, p. $441-448,2009$

17 HUFF-LONERGAN, E.; LONERGAN, S.M. Mechanisms of water-holding capacity of meat: The role of postmortem biochemical and structural changes. Meat Science, v.71, p.194-204, 2005.

18 HUGUES, J.M.; OISETH, S.K.; PURSLOWC, P.P.; WARNER, R.D. A structural approach to understanding the interactions between colour, water-holding capacity and tenderness. Meat Science, v.98, p.520-532, 2014.

19 HUR, S.J.; JIN, S.K.; KIM, I.S. Effect of extra virgin olive oil substitution for fat on quality of pork patty. Journal of the Science of Food and Agriculture, v.88, p.1231-1237, 2008

20 JAYATHILAKAN, J.; SHARMA, G.K.; RADHAKRISHNA, K.; BAWA, A.S. Antioxidant potential of synthetic and natural antioxidants and its effect on warmed-over-flavour in different species of meat. Food Chemistry, v.105, p. 908-916, 2007.

21 JIMÉNEZ-COLMENERO, F. Alimentos funcionais uma oportunidade para o sector cárneo. Associação Nacional dos Industriais de Carnes. ANIC- Gabinete Técnico. Lisboa, n. ${ }^{1 / 2}$, p.15-17, 2006.

22 MARIUTTI, L.R.; BRAGAGNOLO, N. Revisão: Antioxidantes Naturais da Família Lamiaceae. Aplicação em Produtos Alimentícios. Brazilian Journal of Food Technology, v.10, n.2, p. 96-103, 2007.

23 MEILGAARD, M. C.; CARR, B.T.; CIVILLE, G.V. Sensory evaluation techniques (4th ed.). Boca Raton: CRC Press, 2006. $464 \mathrm{p}$

24 MORRISSEY, P.A.; SHEEHY, P.J.A.; GALVIN, K.; KERRY, J.P.; BUCKLEY, D.J. Lipid stability in meat products. Meat Science, v.49, p. 73-86, 1998.

25 MOSKOWITZ, H.R. Product optimization approaches and applications. In: MAcFIE, H.J.; THOMSON, D.M. (Eds.). Measurement of food preferences. London (UK): Blackie Academic and Professional, 1994. p.67-136.

26 MOZAFFARIAN, D.; ASCHERIO, A.; HU, F.B.; STAMPFER, M.J.; WILLETT, W. C.; SISCOVICK, D.S.; RIMM, E.B. Interplay between different polyunsaturated fatty acids and risk of coronary heart disease in men. Circulation, v.111, p.157-164, 2005.

27 NOVELLO, D.; POLLONIO, M.A.R. Adição de linhaça dourada (Linum Usitatissimum L.) e derivados em hambúrgueres bovinos: Aceitação sensorial e análise de sobrevivência. Boletim do CEPPA, v.30, n.2, p. 273-286, 2012. 
28 OLMEDILLA-ALONSO, B.; JIMÉNEZ-COLMENERO, F.; SÁNCHEZ-MUNIZ, F.J. Development and assessment of healthy properties of meat and meat products designed as functional foods. Meat Science, v.95, p.919-930, 2013.

29 O'SULLIVAN, M.G.; BYRNE, D.V.; JENSEN, M.T.; ANDERSEN, H.J.; VESTERGAARD, J. A comparison of warmed-over flavour in pork by sensory analysis, GC/MS and the electronic nose. Meat Science, v. 65, p. 1125-1138, 2003.

30 ÖZVURAL, E. B.; VURAL, H. Utilization of interesterified oil blends in the production of frankfurters. Meat Science, v.78, p.211-216, 2008.

31 PEARCE, K.L.; ROSENVOLD, K.; ANDERSEN, H.J.; HOPKINS, D.L. Water distribution and mobility in meat during the conversion of muscle to meat and ageing and the impacts on fresh meat quality attributes - A review. Meat Science, v.89, p. 111-124, 2011.

32 RUSAK, G.; KOMES, D.; LIKIC, S.; HORZIC, D.; KOVAC, M. Phenolic content and antioxidative capacity of green and white tea extracts depending on extraction conditions and the solvent used. Food Chemistry, v. 110, p. 852-858, 2008.

33 SALCEDO-SANDOVAL, L.; COFRADES, S.; RUIZ-CAPILLAS, C.; CARBALLO, J.; JIMÉNEZ-COLMENERO, F. Konjacbased oil bulking system for development of improved-lipid pork patties: Technological, microbiological and sensory assessment. Meat Science, v. 101, p.95-102, 2015.

34 SAS. Statistical Analysis System. SAS User's guide. V.9.1. Cary, NC. USA: Statistical Analysis Systems Institute,Inc., 2002.

35 SEBRANEK, J.G.; SEWALT, V.J.H.; ROBBINS, K.L.; HOUSER, T.A. Comparison of a natural rosemary extract and BHA/BHT for relative antioxidant effectiveness in pork sausage. Meat Science, v. 69, p. 289-296, 2005.

36 SERDAROGLU, M.; DEGIRMENCIOGLU, O. Effects of fat level $(5 \%, 10 \%, 20 \%)$ and corn flour $(0 \%, 2 \%, 4 \%)$ on some properties of Turkish type meatballs (koefte). Meat Science, v.68, p. 291-296, 2004.

37 SUMAN, S.P.; FAUSTMAN, C.; LEE, S.; TANG, J.; SEPE, H.A.; VASUDEVAN, P.; ANNAMALAI, T.; MANOJKUMAR, M.; MAREK, P.; VENKITANARAYANAN, K.S. Effect of erythorbate, storage and high-oxygen packaging on premature browning in ground beef. Meat Science, v.69, p.363-369, 2005.

38 TARLADGIS, B.G.; WATTS, B.M.; YOUNATHAN, M.T.; DUGGAN, L. A distillation method for the quantitative determination of malonaldehyde in rancid foods. Journal of the American Oil Chemistry Society, v. 37, p 44-48. 1960.

39 TREVINO, J.; RODRIGUEZ, M. L.; ORTIZ, L. T.; REBOLE, A.; ALZUETA, C. Protein quality of linseed for growing broiler chicks. Animal Feed Science and Technology, v.84, p. 155-166, 2000.

40 VALENCIA, I.; ANSORENA, D.; ASTIASARÁN, I. Stability of linseed oil and antioxidants containing dry fermented sausages: A study of the lipid fraction during different storage conditions. Meat Science, v. 73, p. 269-277, 2006.

41 VALÊNCIA, I.; O'GRADY, M.N.; ANSORENA, D.; ASTIASARÁN, I.; KERRY, J.P. Enhancement of the nutritional status and quality of fresh pork sausages following the addition of linseed oil, fish oil and natural antioxidants. Meat Science, v. 80, p. $1046-1054,2008$.

42 YILMAZ, I.; SIMSEK, O.; ISIKLI, M. Fatty acid composition and quality characteristics of low-fat cooked sausages made with beef and chicken meat, tomato juice and sunflower oil. Meat Science, v.62, p. 253-258, 2002.

43 WHO. World and Health Organization. Diet, nutrition and the prevention of chronic diseases, WHO technical report series, n. 916, 2003, 160 p. 